\title{
Environmental measurement in Greenhouse by mobile robot using ROS
}

\author{
Hikaru KUMAMOTO ${ }^{\mathrm{a}}$, Kenichi IIDA ${ }^{\mathrm{a},{ }^{*},}$, Shigeto NAKAMURA ${ }^{\mathrm{a}}$, Etsuko UEDA ${ }^{\mathrm{b}}$ \\ ${ }^{a}$ National Institute of Technology, Nara College, 22 Yata, Yamatokoriyama, Nara, 639-1080, Japan

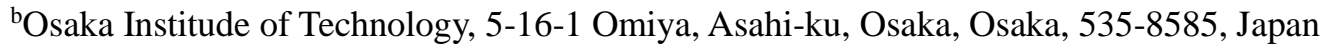 \\ *Corresponding Author: iida@ctrl.nara-k.ac.jp
}

\begin{abstract}
The environmental information in the greenhouse is one of the essential items in precision agriculture for improving the productivity of crops. In many cases of environmental measurement in the greenhouse, a fixed sensor can measure near the installation location. However, to estimate the distribution of the environmental information in the whole greenhouse, it is necessary to install many sensors, which increases the cost. Therefore, in this study, to acquire more the detailed environmental information, we propose the system to measure the environmental information in the greenhouse by the mobile robot using ROS. The proposed mobile robot consists of the $\mathrm{CO}_{2}$ sensor, the temperature sensor, the humidity sensor and LRF for environmental sensing. In this paper, we confirmed the usefulness of the proposed system from the verification experiment using a prototype mobile robot.
\end{abstract}

Keywords: Agricultural support, Mobile robot, ROS.

\section{Introduction}

For sustainable agricultural growth, a realization of efficient and stable agricultural management ${ }^{(1)}$ is required. Therefore, Ministry of Agriculture, Forestry and Fisheries promotes the precision agriculture based on environmental information in farmland to control the production of crops without depending on experience and intuition, and the smart-agriculture using advanced technologies such as $\mathrm{AI}^{(2)}$, IoT $^{(3-6)}$, $\operatorname{robots}^{(7-11)}$, and so on. Moreover, there have been many studies ${ }^{(12-16)}$ about environmental measurement so that cultivating crops based on quantitative environmental information. Generally, many studies acquire the environmental data from fixed environmental sensors on farmland, for example, Environmental Sensing in a Plastic
Greenhouse by Sensor Network ${ }^{(17)}$ and Development of Field Servers for a Field Monitoring System ${ }^{(18)}$.

However, the measuring range of fixed sensors is restricted. Thus, it is difficult to know the detailed information in the whole greenhouse. Consequently, in the case of the large-scale greenhouse, many sensors are required, hence the cost increases.

In this study, we propose the more detailed environmental measurement system by automatic patrol using a mobile robot equipped with the environmental sensor unit, assuming the large-scale greenhouse.

\section{The proposed system}

\subsection{System configuration}

Fig. 1 shows the outline of the proposed system. The mobile robot acquires the detailed environmental information by automatic patrol between the benches in the elevated cultivation greenhouse. Furthermore, the obtained environmental information can be used by farmers as the environmental map in the greenhouse.

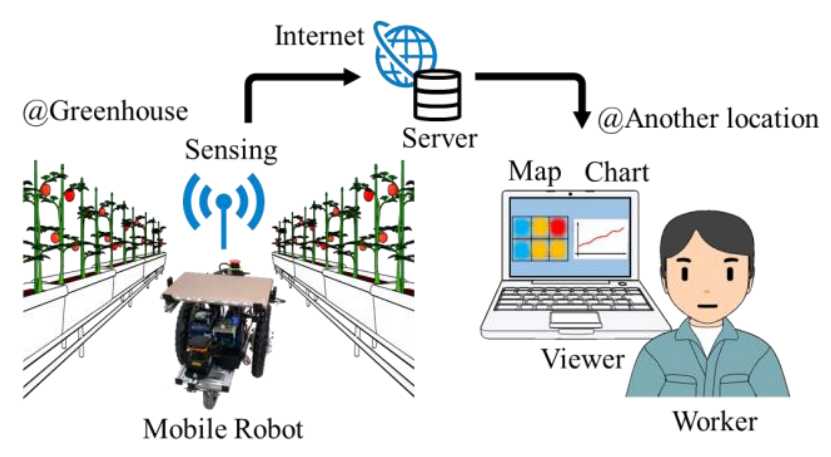

Fig. 1. Outline of proposed system. 
Fig. 2 and Fig. 3 show the overview of the mobile robot and the system configuration. The mobile robot applies the differential two-wheeled robot consists of two wheels and a caster attached to the front. Also, the environmental sensor unit for environmental sensing in the greenhouse as shown in Fig. 4 equips with a $\mathrm{CO}_{2}$ sensor, temperature sensor, and humidity sensor. Furthermore, the robot drive unit provides with DC motors, motor drivers and rotary encoders on the left and right wheels for driving and reading the rotation velocity as shown in Fig. 5. Besides, the mobile robot while patrols in the greenhouse, provides with the laser rangefinder (LRF) for the acquisition of layout and the obstacle information. The mobile robot run on the robot operating system (ROS) which is a framework in robot development ${ }^{(19}$, 20). Tables 1-4 show the specifications of the mobile robot, the environmental sensors, the motor with the encoder, and the LRF respectively.

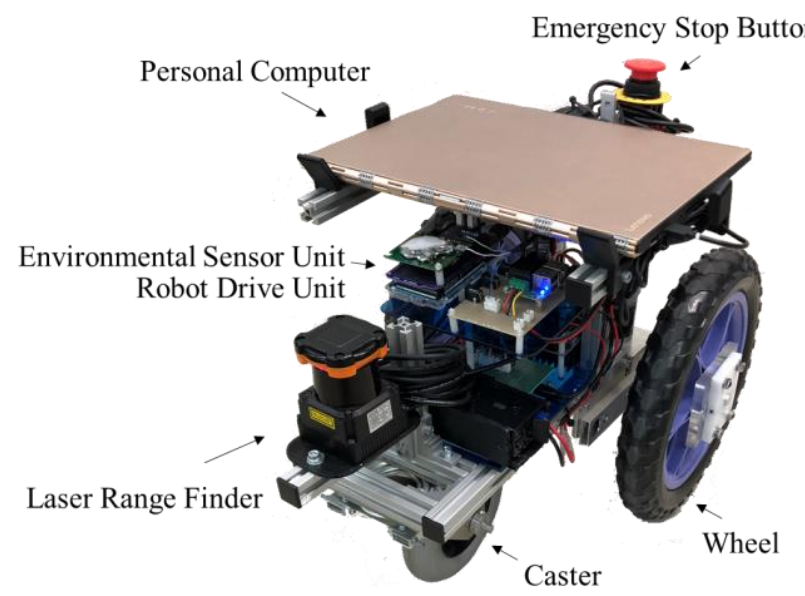

Fig. 2. Overview of the mobile robot.

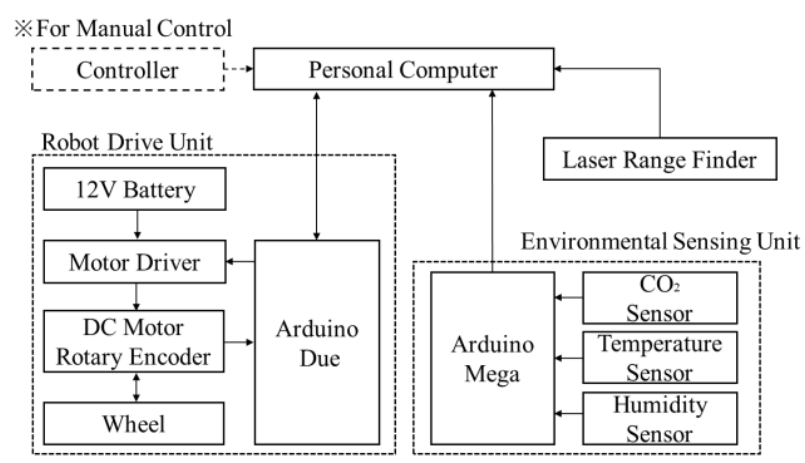

Fig. 3. System configuration.
Table 1. Specification of the mobile robot.

\begin{tabular}{|l|c|}
\hline Size $(\mathrm{W} \times \mathrm{D} \times \mathrm{H})[\mathrm{mm}]$ & $300 \times 500 \times 450$ \\
\hline Tread Width $[\mathrm{mm}]$ & 292 \\
\hline Wheel Diameter $[\mathrm{mm}]$ & 294 \\
\hline Weight $[\mathrm{kg}]$ & 7.8 \\
\hline Payload $[\mathrm{kg}]$ & 2.2 \\
\hline Maximum Speed $[\mathrm{km} / \mathrm{h}]$ & 7.2 \\
\hline
\end{tabular}

Table 2. Specification of the environmental sensors.

\begin{tabular}{|l|c|c|c|}
\hline & Range & Accuracy & Resolution \\
\hline $\mathrm{CO}_{2}[\mathrm{ppm}]$ & $0 \sim 2000$ & \pm 30 & 1.95 \\
\hline Temperature $\left[{ }^{\circ} \mathrm{C}\right]$ & $-40 \sim+85$ & \pm 1 & 0.01 \\
\hline Humidity $[\%]$ & $0 \sim 100$ & \pm 3 & 0.008 \\
\hline
\end{tabular}

Table 3. Specification of the motors with encoders.

\begin{tabular}{|l|c|}
\hline Motor Rated Voltage [V] & 18 \\
\hline Motor Rated Revolution [rpm] (No Load) & $250(219)$ \\
\hline Encoder Resolution [ppr] & 360 \\
\hline
\end{tabular}

Table 4. Specification of the LRF.

\begin{tabular}{|l|c|}
\hline Range & 0.1 to $30[\mathrm{~m}], 270\left[^{\circ}\right]$ \\
\hline Accuracy & 0.1 to $10[\mathrm{~m}]: \pm 30[\mathrm{~mm}]$ \\
\cline { 2 - 2 } & 10 to $30[\mathrm{~m}]: \pm 50[\mathrm{~mm}]$ \\
\hline Angular Resolution & $0.25\left[^{\circ}\right]\left(270\left[{ }^{\circ}\right] / 1080[\mathrm{steps}]\right)$ \\
\hline
\end{tabular}

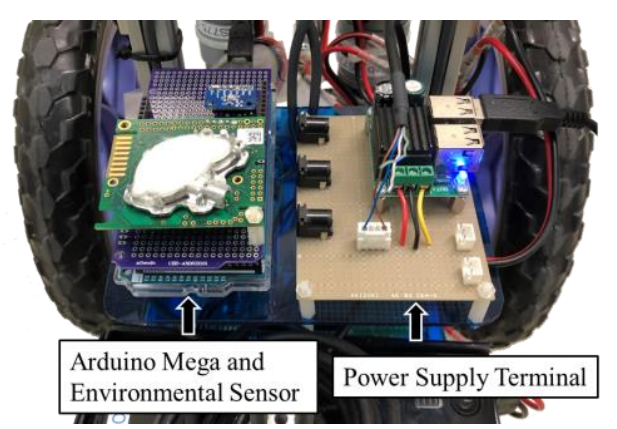

Fig. 4. Overview of environmental sensor unit.

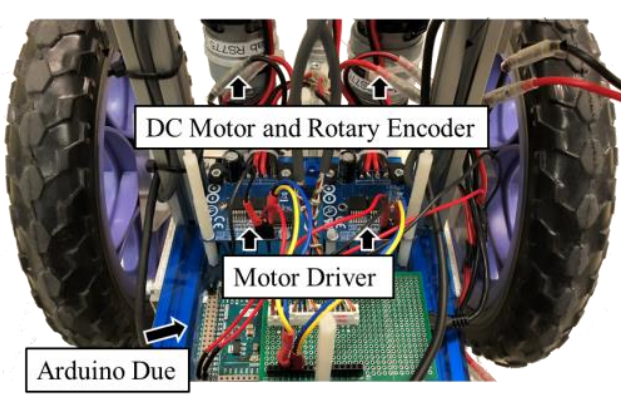

Fig. 5. Overview of robot drive unit. 


\subsection{Patrolling mobile robot using ROS}

Automatic patrol using the proposed system is conducted by the following two procedures.

Procedure 1: Creation of the map.

The operator manually operates the mobile robot to acquire the layout information in the greenhouse measured by the LRF and the odometry of the mobile robot. The obtained information creates a map necessary for the mobile robot to travel autonomously using the "slam_gmapping stack" of ROS.

Procedure 2: Setting of the route of the patrol.

Based on the map acquired in procedure 1, the operator set multiple target points on the route for measuring environmental information. The mobile robot becomes possible to patrol by repeating the autonomous traveling to each setting target points. Now, the mobile robot to move autonomously adopt "navigation stack" of ROS.

Fig. 6 shows the configuration of the ROS packages for the proposed patrol system. The function of the ROS packages is described below. Firstly, "navigation" consists of three packages which are "move_base", "map_server", and "amcl" (Adaptive Monte Carlo Localization). "move_base" is a framework of autonomous movement, and it consists of "global_planner", "local_planner", “global_costmap" and "local_costmap" for management of obstacle, "recovery_behaviors" for robot stack status, to be possible to move the robot to the target point on the created map. "map_server" provides map information to "move_base" and "amcl". Then, "amcl" performs localization with scan data from the LRF and the odometry of the robot. The proposed system is introduced "urg_node" which is a driver for acquiring scan data from the LRF, "tf" transform coordinate frames of map, mobile robot, and the LRF, and "rosserial" which is a protocol for serial devices such as Arduino. Furthermore, the proposed system is introduced

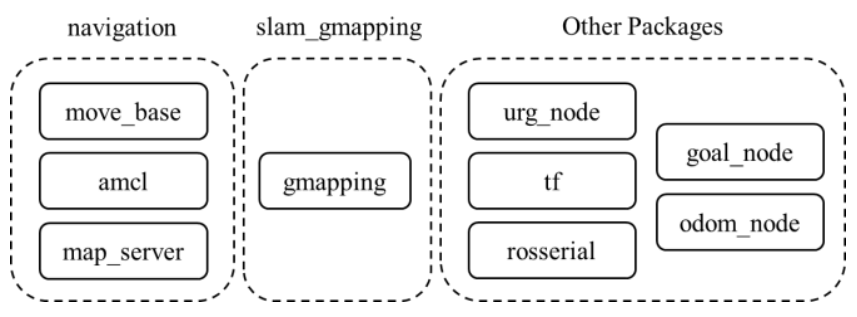

Fig. 6. Configuration of the ROS packages. "odom_node" of own development for estimation the moving amount of the mobile robot. After that, "odom_node" provides the odometry of the mobile robot to "move_base" and "amcl" from kinematics of two-wheeled robot as shown in Fig. 7. In this figure, $r$ is the turning radius, $v$ is the straight traveling velocity, $w$ is the angular velocity, $d$ is the half of the tread width, $v_{r}$ and $v_{l}$ are the right and left wheels velocities. The geometric relationships between every parameters in Fig. 7 are shown by Equations (1) and (2).

$$
\left.\begin{array}{l}
r=v / w \\
v_{r}=(r+d) w \\
v_{l}=(r-d) w
\end{array}\right\}
$$

As the amount of movement of the robot, the straight traveling velocity $v$ and the angular velocity $w$ are estimated by Equation (3).

$$
\left.\begin{array}{c}
v=\left(v_{r}+v_{l}\right) / 2 \\
w=\left(v_{r}-v_{r}\right) / 2 d
\end{array}\right\}
$$

Also, the setting of the target points using "navigation" generally performs by the GUI on visualization tool "rviz". However, the GUI on visualization tool "rviz" can not to set sub-goal on the route to the final destination. Therefore, it is possible to set a sub-goal on the route to the final destination by "goal_node" of own development in the proposed system, it is possible to arbitrarily design the route of the patrol.

\subsection{Presentation of environmental information}

The measured environmental information by patrolling using a mobile robot is the data on the coordinates of each measurement point, and it is difficult for the farmer to understand the conditions in the whole greenhouse. Thus, to present the case in the entire greenhouse to farmer more effectively, we create an environmental map which overlays the greenhouse layout on the measured environmental information.

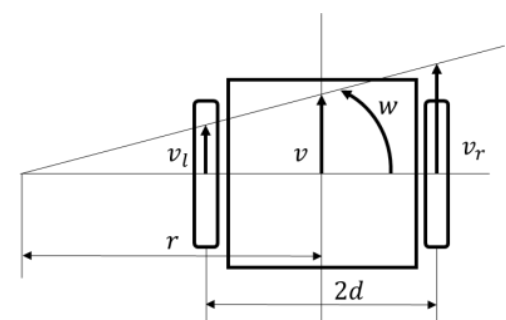

Fig. 7. Kinematics of two-wheel robot. 
Fig. 8 shows the visualization flow of environmental information. The environmental map is generated by executing a python script with the 2-D occupancy grid map and integrated environmental information and traveling locus on ROS. The environmental data is shown in the greenhouse layout. Hence farmer easily can understand the conditions in the whole greenhouse.

\section{Evaluation experiment}

To verify the usefulness of the proposed system, we conducted an environmental measurement experiment by patrolling using the mobile robot at the actually elevated cultivation greenhouse as shown in Fig. 9. Fig. 10 shows the layout of the greenhouse. In the figure, (1)-(5) are the target points and the blue arrows show the traveling route. In addition, the mobile robot travels at approximately $0.17 \mathrm{~m} / \mathrm{s}$ and performs environmental measurement every second. Hence the environmental data obtain the same as measured using approximately 58 fixed sensors every $10 \mathrm{~m}$. As shown in Fig. 11, the mobile robot traveled and acquired the layout in the whole greenhouse.

Fig. 12 shows the 2-D occupancy grid map generated using "slam_gmapping", and it confirmed that the actual layout of the greenhouse had been reproduced. Fig. 13 and Fig. 14 show the environmental maps (the temperature and the humidity) with the environmental information measured on the travel locus, respectively. As shown in the figure, the environmental measurement points are indicated on the actual layout of the greenhouse, and it is possible to confirm the distribution of the temperature and the humidity. Also, in this experiment, about 500 environmental measurement points were acquired by once patrol. Hence it has confirmed that the detailed measurement of environmental information is possible. Furthermore, it is possible to adjust the measurement points, e.g., arbitrarily position of the measurement points by patrolling route, the number of the measurement points by robot velocity or measurement

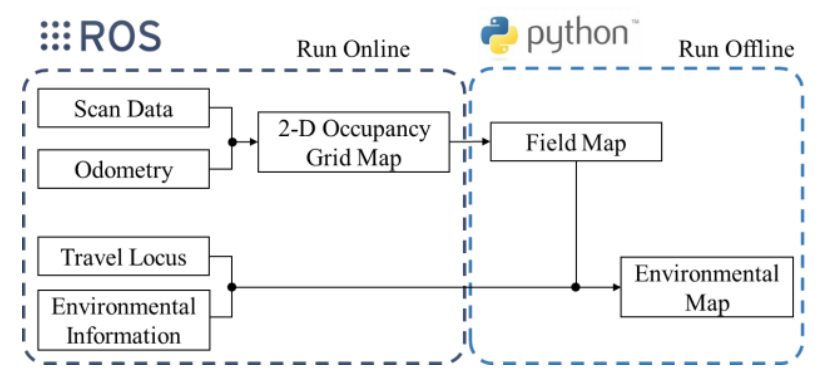

Fig. 8. Visualization flow of environmental information sampling rate. Fig. 15 and Fig. 16 show the interpolation maps by inverse distance weight (IDW) calculated Equation (4), (5) from the obtained environmental data.

$$
u(x)=\frac{\sum_{i=0}^{N} w_{i}(x) u\left(x_{i}\right)}{\sum_{j=0}^{N} w_{j}(x)}
$$

$$
w_{i}(x)=\frac{1}{d\left(x, x_{i}\right)^{p}}
$$

Where $x$ is estimated points, $u(x)$ and $w_{i}(x)$ are each characteristic value, $d\left(x, x_{i}\right)$ is distance between two points, $p$ is distance index.

As shown in the figure, the measured point group data is converted to planar information by interpolation. Hence the farmer can visually confirm the distribution of the detailed environmental information of the left side of the greenhouse. Also, more precise interpolation is possible by setting the traveling route in detail. By contrast, the right side of the greenhouse cannot be confirmed the distribution of the detailed environmental information because of using only a fixed sensor data. Therefore, the proposed system by the mobile robot can acquire more the detailed environmental data in the greenhouse.

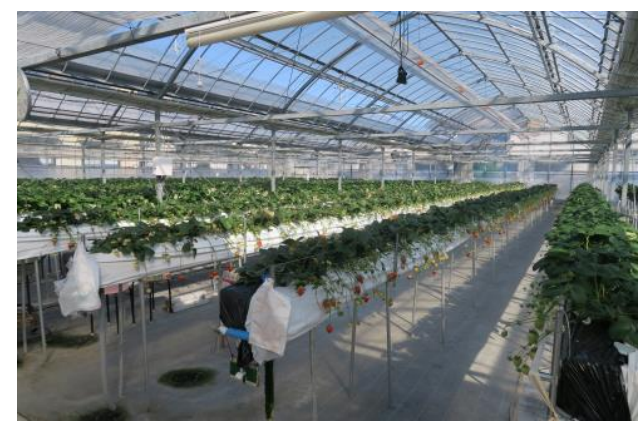

Fig. 9. Overview of the greenhouse.

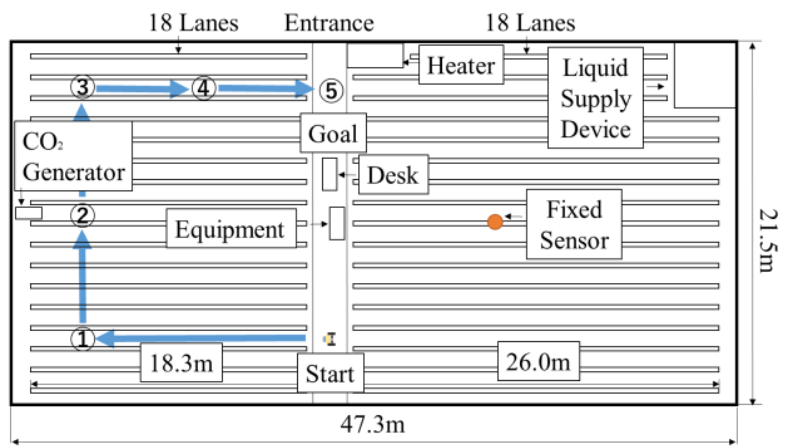

Fig. 10. Condition of the greenhouse. 


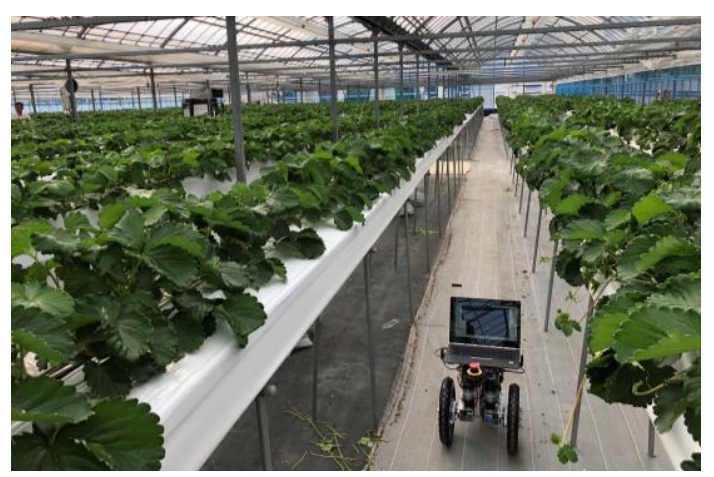

Fig. 11. Movement of mobile robot.

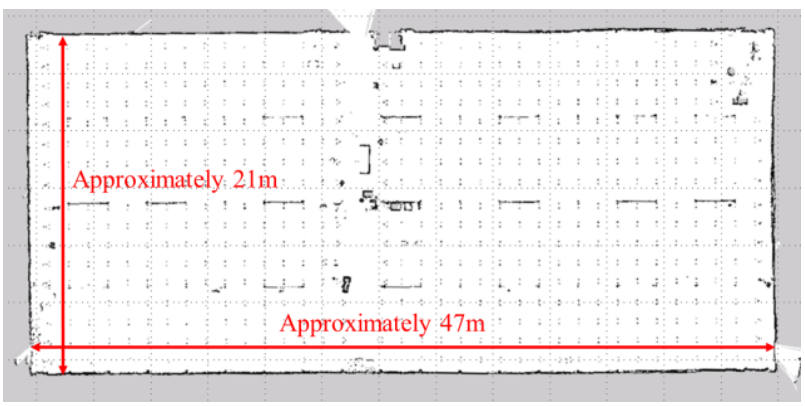

Fig. 12. Generated map.

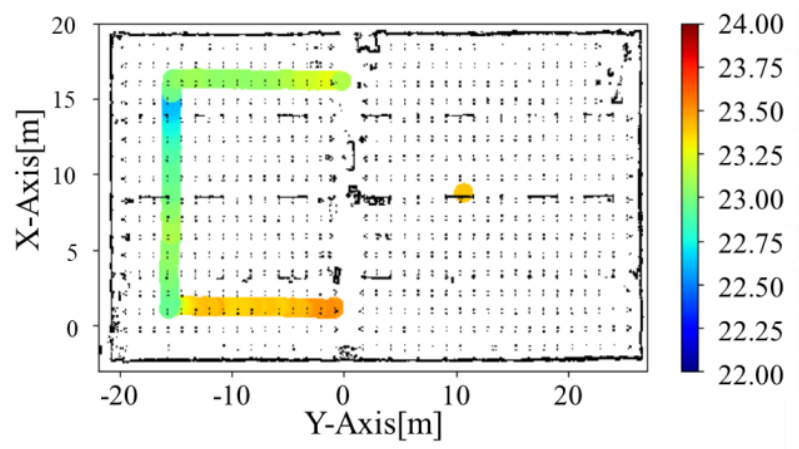

Fig. 13. Environmental map (Temperature).

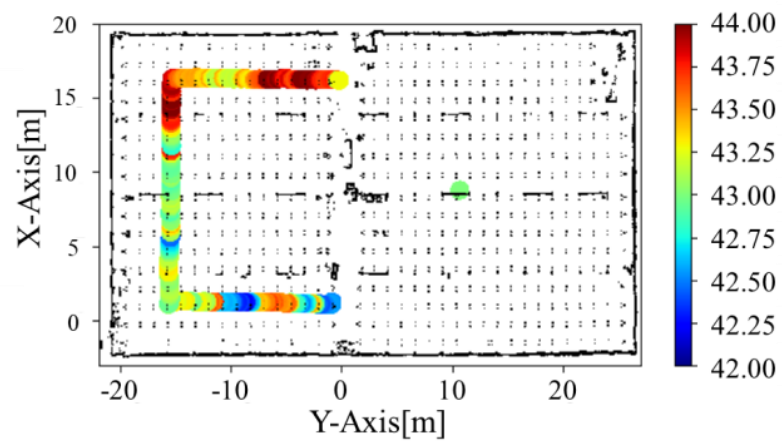

Fig. 14. Environmental map (Humidity).

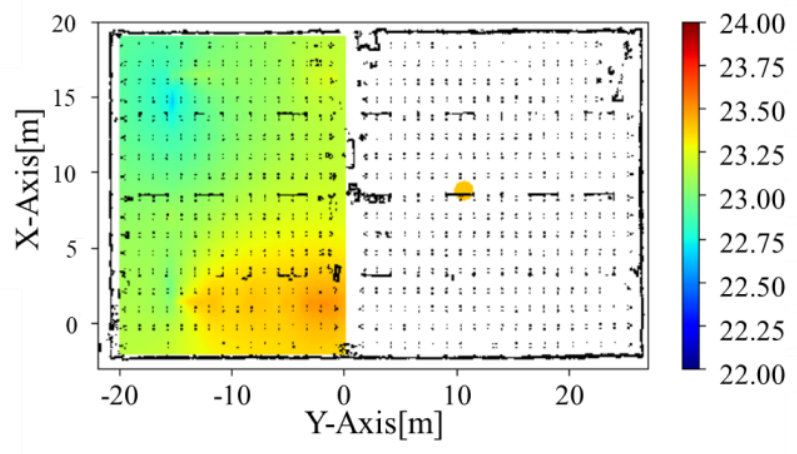

Fig. 15. Interpolation map using IDW (Temperature).

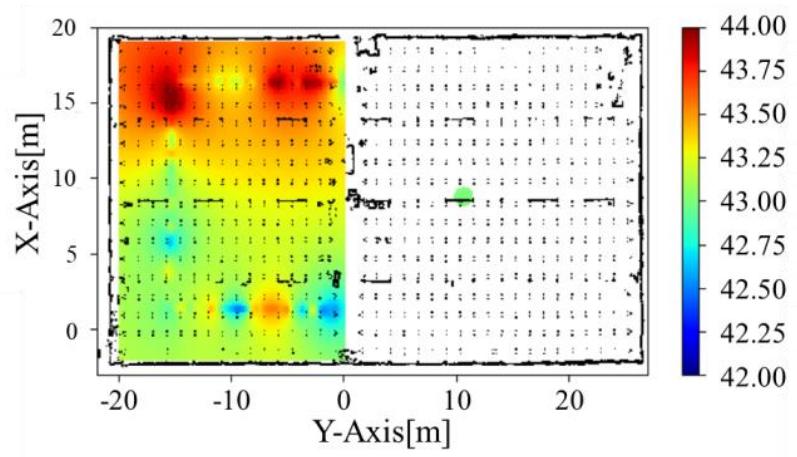

Fig. 16. Interpolation map using IDW (Humidity).

\section{Conclusions}

In this study, we proposed more the detailed environmental measurement system in the greenhouse by patrolling using the mobile robot. Furthermore, the experimental results in the greenhouse demonstrated that the mobile robot could patrol designated routes while measuring the environmental information. The mobile robot equipped with the sensor unit acquired many environmental measurement data. Hence the proposed system can obtain more the detailed environmental data in the greenhouse. Although it is desirable to patrol all lanes at 24 hours a day ideally, indeed it takes time cost depends on the scale of the greenhouse. Therefore, it is necessary to consider the optimum measurement frequency and area. Furthermore, the proposed method can be applied to measure the full environmental distribution in factories and offices. We are planning to the improvement of the proposed system so that farmer can more easily handle the measured environmental information, such as IoT cloud service and completely automatically sensing to discuss. 


\section{References}

(1) "Annual Report on Food, Agriculture and Rural Areas trends in Japan FY 2017", The ministry of Agriculture, Forestry and Fisheries of Japan, p. 1, 2017

(2) Shumpei Uegaki, Hidekazu Araki, Ryo Toshima, Makoto Shinzaki, Daisuke Ueta, and Ryuji Yamazaki : "Tomato-Harvesting Robot using AI for Environment Recognition”, Panasonic Technical Journal, Vol. 64, No. 1, pp. 54-59, 2018

(3) Karim Foughali, Karim Fathallah, and Ali Frihida : "Using Cloud IOT for ddisease prevention in precision agriculture", Procedia Computer Science, Vol. 130, pp. 575-582, 2018

(4) Mohanraj I , Kirthika Ashokumar, and Naren J : "Field Monitoring and Automation using IOT in Agriculture Domain”, Procedia Computer Science, Vol. 93, pp. 931939, 2016

(5) Mustafa Alper Akkaşa, and Radosveta Sokullu : “An IoT-based greenhouse monitoring system with Micaz motes", Procedia Computer Science", Vol. 113, pp. 603608, 2017

(6) Foughali Karim, Fathalah Karim, and Alifrihida : "Monitoring system using web of things in precision agriculture", Procedia Computer Science”, Vol. 109, pp. 1104-1109, 2016

(7) Juha Backman, Pyry Piirainen, and Timo Oksanen : "Smooth turning path generation for agricultural vehicles in headlands", Biosystems Engineering, Vol. 139, pp. 76-86, 2015

(8) JosseDe Baerdemaker : "Precision Agriculture Technology and Robotics for Good Agricultural Practices", IFAC Proceedings Volumes, vol. 46, No. 4, pp. 1-4, 2013

(9) Philip J.Sammons, Tomonari Furukawa, and Andrew Buligin : "Autonomous Pesticide Spraying Robot for use in a Greenhouse", pp. 1-9, 2005

(10) Gilad Gat, Samuel Gan-mor, and Amir Degani : " Stable and robust vehicle steering control using an overhead guide in greenhouse tasks", Computers and Electronics in Agriculture", Vol. 121, pp. 234-244, 2016

(11) Simon János : "WSN implementation in the Greenhouse Environment Using Mobile Measuring Station", International Journal of Electrical and Computer Engineering Systems, Vol. 1, No. 1, pp. 37-44, 2010

(12)I.-Chang Yang, Kuang-Wen Hsieh, Chao-Yin Tsai, Yu-I. Huang, Yu-Liang Chen, and Suming Chen : "Development of an automation system for greenhouse seeding production management using radio-frequencyidentification and local remote sensing techniques", Engineering in Agriculture, Environment and Food, Vol.

7, No.1, pp. 52-58, 2014

(13) Javier López-Martínez, José L.Blanco-Claraco, José Pérez-Alonso, and Ángel J.Callejón-Ferre : "Distributed network for measuring climatic parameters in heterogeneous environments: Application in a greenhouse", Computers and Electronics in Agriculture, Vol. 145, pp. 105-121, 2018

(14)B.Marchial, S.Zanonia, and M.Pasetti : "Industrial Symbiosis for Greener Horticulture Practices: The $\mathrm{CO} 2$ Enrichment from Energy Intensive Industrial Processes", Procedia CIRP, Vol. 69, pp. 562-567, 2018

(15) Prashant Singh Chauhan, Anil Kumar, and Chayut Nuntadusit : "Heat transfer analysis of PV integrated modified greenhouse dryer", Renewable Energy, Vol. 121, pp. 53-65, 2018

(16) J.del Sagrado, J.A.Sánchez, F.Rodríguez, and M.Berenguel : "Bayesian networks for greenhouse temperature control", Journal of Applied Logic, Vol. 17, pp. $25-35,2016$

(17) Takayuki Suyama, Futoshi Naya, and Yutaka Yanagisawa : "Environmental Sensing in a Plastic Greenhouse by Sensor Network", DICOMO2013, pp. 938-944, 2013

(18) Tokihiro Fukatsu, and Masayuki Hirafuji : "Development of Field Servers for a Field Monitoring System”, Agricultural Information Research, Vol. 12, pp. $1-12,2013$

(19) Yoshitaka Hara : "Autonomous navigation with ROS", Journal of the Robotics Society of Japan, Vol. 35, No.4, pp. 286-290, 2017

(20) Atsushi Watanabe : “ Development of a ROS-based Oil Plant Inspection Mobile Robot System - Making Decision between ROS Ecosystem's and From-Scratch Packages - Journal of the Robotics Society of Japan, Vol. 35, No.4, pp. 291-294, 2017 